University of Nebraska - Lincoln

DigitalCommons@University of Nebraska - Lincoln

$11-1998$

\title{
Ionization Dynamics in Fast Ion-Atom Collisions. I. Energy and Angular Distributions of Low-Energy Electrons Emitted in Ionization of He by Bare Carbon lons
}

\author{
Lokesh C. Tribedi \\ Tata Institute of Fundamental Research, Homi Bhabha Road, Colaba, Mumbai 400005, India \\ P. Richard \\ J. R. Macdonald Laboratory, Department of Physics, Kansas State University, Manhattan, Kansas \\ Y. D. Wang \\ J. R. Macdonald Laboratory, Department of Physics, Kansas State University, Manhattan, Kansas \\ C. D. Lin \\ J. R. Macdonald Laboratory, Department of Physics, Kansas State University, Manhattan, Kansas \\ L. Gulyas \\ Institute of Nuclear Research of the Hungarian Academy of Science (ATOMKI), P.O. Box 51, H-4001 \\ Debrecen, Hungary
}

See next page for additional authors

Follow this and additional works at: https://digitalcommons.unl.edu/physicsrudd

Part of the Physics Commons

Tribedi, Lokesh C.; Richard, P.; Wang, Y. D.; Lin, C. D.; Gulyas, L.; and Rudd, M. Eugene, "Ionization Dynamics in Fast Ion-Atom Collisions. I. Energy and Angular Distributions of Low-Energy Electrons Emitted in Ionization of He by Bare Carbon Ions" (1998). M. Eugene Rudd Publications. 27.

https://digitalcommons.unl.edu/physicsrudd/27

This Article is brought to you for free and open access by the Research Papers in Physics and Astronomy at DigitalCommons@University of Nebraska - Lincoln. It has been accepted for inclusion in M. Eugene Rudd Publications by an authorized administrator of DigitalCommons@University of Nebraska - Lincoln. 


\section{Authors}

Lokesh C. Tribedi, P. Richard, Y. D. Wang, C. D. Lin, L. Gulyas, and M. Eugene Rudd 


\title{
Ionization dynamics in fast ion-atom collisions. I. Energy and angular distributions of low-energy electrons emitted in ionization of He by bare carbon ions
}

\author{
Lokesh C. Tribedi, ${ }^{1, *}$ P. Richard, ${ }^{2}$ Y. D. Wang, ${ }^{2}$ C. D. Lin, ${ }^{2}$ L. Gulyas, ${ }^{3}$ and M. E. Rudd ${ }^{4}$ \\ ${ }^{1}$ Tata Institute of Fundamental Research, Homi Bhabha Road, Colaba, Mumbai 400005, India \\ ${ }^{2}$ J. R. Macdonald Laboratory, Department of Physics, Kansas State University, Manhattan, Kansas 66506-2601 \\ ${ }^{3}$ Institute of Nuclear Research of the Hungarian Academy of Science (ATOMKI), P.O. Box 51, H-4001 Debrecen, Hungary \\ ${ }^{4}$ Department of Physics and Astronomy, University of Nebraska-Lincoln, Lincoln, Nebraska 68588-0111
}

(Received 20 April 1998)

\begin{abstract}
We have studied the two-center effect (TCE) in ion-atom ionization by measuring the energy and angular distributions of the double-differential cross sections (DDCSs) $\left(d^{2} \sigma / d \varepsilon d \Omega\right)$ of the low-energy electrons emitted in a collision of $\mathrm{He}$ atoms with $2.5 \mathrm{MeV} /$ nucleon $\mathrm{C}^{6+}$ ions. The electrons with energies between 0.1 and $300 \mathrm{eV}$ were detected for 13 different emission angles between $15^{\circ}$ and $160^{\circ}$. From the measured DDCSs we have deduced the single differential cross sections such as $d \sigma / d \Omega$ and $d \sigma / d \varepsilon$ and the total cross section. The data have been compared with the continuum distorted wave eikonal initial state calculations with $\mathrm{H}$-like and Hartree-Fock-Slater wave functions for the initial and final state of the electron and first Born calculations. The forward-backward asymmetry parameter also has been deduced to study the TCE in detail. In the following paper [Lokesh C. Tribedi et al., Phys. Rev. A 58, 3626 (1998)] we have explored the collision dynamics by deducing the momentum distributions of the electron, the recoil ion, and the projectile.

[S1050-2947(98)04810-0]

PACS number(s): 34.50.Fa
\end{abstract}

\section{INTRODUCTION}

Ionization is one of the most important reactions in highenergy ion-atom collisions. The low-energy electrons are emitted with the largest probability. The measurements of these electrons' cross sections differential in energy and emission angle could provide crucial information on ionization dynamics. The richness of the field originates from the possibility of studying the dynamics of an ionized or free (with momentum analyzed) electron in the presence of two moving sources of Coulomb potentials. The early measurements on the electron double-differential cross sections (DDCSs) involve mostly the low-charged projectiles such as electrons, protons, and $\mathrm{He}$ ions [1-9]. There have only been a few measurements on electron DDCSs using partially stripped or bare ions [10-15]. The mechanism of ionization by highly charged ions is not completely understood. The electrons emitted in ionization are simultaneously influenced by the long-range Coulomb fields of the target and the projectile. Such two-center effects and the postcollision interaction play a major role in the case of ionization by highly charged ions. The energy and angular distributions of the electrons in highly charged ion induced ionization provide a fertile field to study the two-center effects.

The first Born approximation (B1) cannot describe the two-center electron emission (TCEE) even at relatively high projectile velocity. The theoretical method based on the continuum distorted wave eikonal initial state (CDW-EIS) approximation has been developed [16] to explain the twocenter effect on electron emission. This method is first order in the distorted wave series and is shown to be adequate to

*Electronic address: lokesh@tifrc3.tifr.res.in describe the dynamics of the ionized electron in the combined Coulomb fields of the projectile and the target. The model was extended to multielectronic targets by Fainstein et al. $[17,18]$. In an independent-particle picture RoothianHartree-Fock [19] and screened H-like orbitals were applied for the initial and final channels, respectively. Recently, the model was further improved [20] by applying Hartree-FockSlater (HFS) wave functions for the initial and final states of the electron being ionized. So the method combines two basic requirements necessary for an adequate description of electron emission in ion-atom collisions: the two-center effects and realistic (numerical) target wave functions. The latter is important to account for electron ejection in the backward directions $[3,20]$. A discrepancy between the DDCS data and CDW-EIS $(\mathrm{H})$ approximation $(\mathrm{H}$ denotes a $\mathrm{H}$-like wave function in the initial state) has been observed [15] for electrons emitted in backward directions in the ionization of molecular hydrogen by bare carbon ions. It is not clear whether molecular effects may cause such a discrepancy. Helium is the simplest two-electron atom used to study ionatom collisions. Stolterfoht et al. [14] have reported the electron DDCS measurements in the ionization of He by different high-energy (5 MeV/nucleon) bare ions. A discrepancy between the CDW-EIS(HFS) prediction and the experimental data remains for cross sections of low- as well as highenergy electrons for different high-energy ions. Such measurements for highly charged ions at lower energy are required for which the two-center effects are expected to be stronger. Fainstein et al. [21] also have applied the CDWEIS model to study the angular asymmetry of the low-energy electrons and have found a large disagreement with the data of Suarez et al. [6] for $p+\mathrm{Ne}$.

In the present paper we provide a stringent test for these theoretical methods via the detailed measurements of the energy and angular distributions of the low-energy electrons 
(0.1-300 eV) emitted in the ionization of He bombarded by bare carbon ions. The data are compared with the B1 and CDW-EIS calculations using the H-like and HFS wave functions. The forward-backward asymmetry also have been studied by comparing the asymmetry parameter with B1 and CDW-EIS calculations. For the present collision system the double ionization contributes only about $5 \%$ (see the following paper, henceforth referred to as paper II, for references) of the single-ionization cross section. Since the majority of the electrons are produced in single ionization, a three-body kinematics has been used in paper II to derive the longitudinal momentum distributions of the electrons, the recoil ions, and the projectiles from the measured electron DDCSs.

\section{EXPERIMENTAL DETAILS}

The details of the experimental technique can be found in Refs. $[7,15]$. The scattering chamber and the spectrometer were built at the University of Nebraska-Lincoln and moved to Kansas State University (KSU). In brief, bare C ions of energy $2.5 \mathrm{MeV} /$ nucleon were obtained from the tandem van de Graaff accelerator at KSU. A hemispherical electrostatic analyzer was used and a small preacceleration voltage $(5 \mathrm{~V})$ was applied on the front aperture at the entrance of the analyzer in order to increase the collection efficiency of the lowenergy electrons. The energy-analyzed electrons were detected by a channel electron multiplier (CEM) mounted on the exit port or aperture of the analyzer. The cone of the CEM was biased at $100 \mathrm{~V}$ to help the low-energy electrons reach the detector.

The spectrometer could be rotated between $15^{\circ}$ and $160^{\circ}$ and the electrons were detected at 13 different angles: $15^{\circ}$, $30^{\circ}, 45^{\circ}, 60^{\circ}, 75^{\circ}, 80^{\circ}, 85^{\circ}, 90^{\circ}, 95^{\circ}, 105^{\circ}, 120^{\circ}, 135^{\circ}$, and $160^{\circ}$. At each angle the data were collected in very fine energy steps for $\varepsilon_{e}$ varying between 0.1 and $300 \mathrm{eV}$. For each angle the spectrum was taken with and without gas in the chamber. The data without gas were used for background subtraction. The chamber was flooded with $\mathrm{He}$ gas at a low pressure $(0.1-0.15 \mathrm{mT})$ for the low-energy scan $(0.1-50 \mathrm{eV})$ in order to minimize the scattering of the low-energy electrons emitted in the ionization of the target. Higher-energy $(30-300 \mathrm{eV})$ scans were made at higher gas pressure $(0.3-$ $0.45 \mathrm{mT}$ ). The pressure dependence was also studied to ascertain the region for single collision conditions. The data were corrected to account for the loss due to the scattering of low-energy electrons from the He gas, but the correction factor was found to be less than $5 \%$ [22].

To put the measured electron yields on an absolute scale, we measured at different angles the electron energy spectrum from the ionization of $\mathrm{He}$ in a collision with $1.5-\mathrm{MeV}$ protons for which the cross section data are known [4]. From these measurements the normalization factor was obtained, which was energy and angle independent within about $7 \%$. The statistical error was low $(<5-10 \%)$ except for the largest angles for which the cross sections are very low. For these angles $\left(\theta_{e} \geqslant 120^{\circ}\right)$ the statistical error was $5-15 \%$. The absolute errors in the cross sections that were typically $20-25 \%$ between 5 and $100 \mathrm{eV}$ resulted from the normalization procedure and the counting statistics. For electron energies below $5 \mathrm{eV}$ and above $100 \mathrm{eV}$ the absolute errors could be as large as $30-50 \%$. The lowest-energy electrons

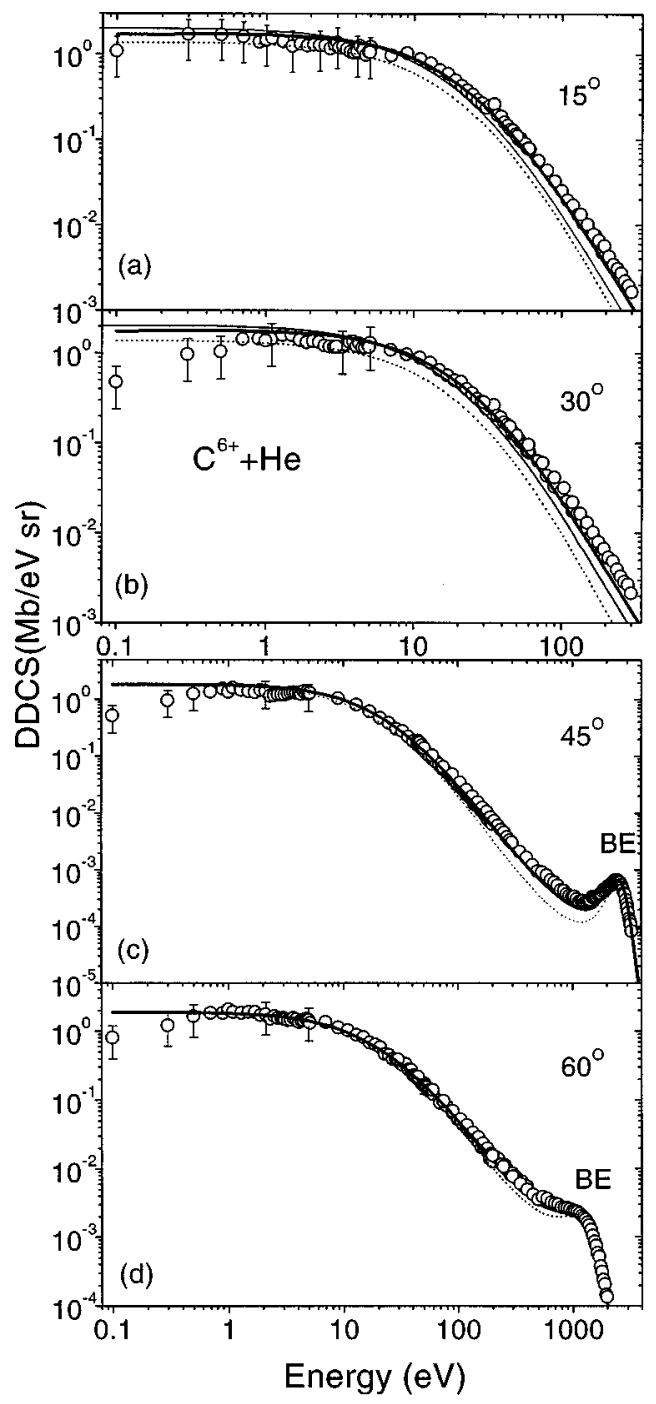

FIG. 1. (a)-(d) Double-differential cross sections of electron emission for four different angles, namely, $\theta=15^{\circ}, 30^{\circ}, 45^{\circ}$, and $60^{\circ}$. The CDW-EIS(HFS), CDW-EIS(H) and B1 calculations are shown by thick solid, thin solid, and dotted lines, respectively.

easily could be deflected by stray fields and may cause additional systematic errors. Extreme precautions were taken to ensure the cleanliness inside the scattering chamber to remove any source of electrostatic fields. The magnetic field was reduced to about $5 \mathrm{mG}$ or less by using $\mu$-metal shielding and an external coil. These were required to detect the lowest-energy electrons $(<1 \mathrm{eV})$. Above $100 \mathrm{eV}$ the cross sections, being too small, had large statistical errors.

\section{RESULTS AND DISCUSSION}

\section{A. Energy dependence at a fixed angle}

In Figs. 1, 2, and 3 we display the electron DDCS spectrum obtained for small forward angles $\left(\theta \leqslant 60^{\circ}\right)$, forward angles $\left(75^{\circ} \leqslant \theta \leqslant 90^{\circ}\right)$, and backward angles $\left(95^{\circ} \leqslant \theta\right.$ $\leqslant 160^{\circ}$ ), respectively. The measured DDCSs for various angles and for some selected energies are shown in Table I. A drop in the cross sections below $0.5 \mathrm{eV}$ could be due to the residual stray fields. The binary encounter (BE) peaks are also shown for $45^{\circ}, 60^{\circ}$, and $75^{\circ}$. The dotted line represents 


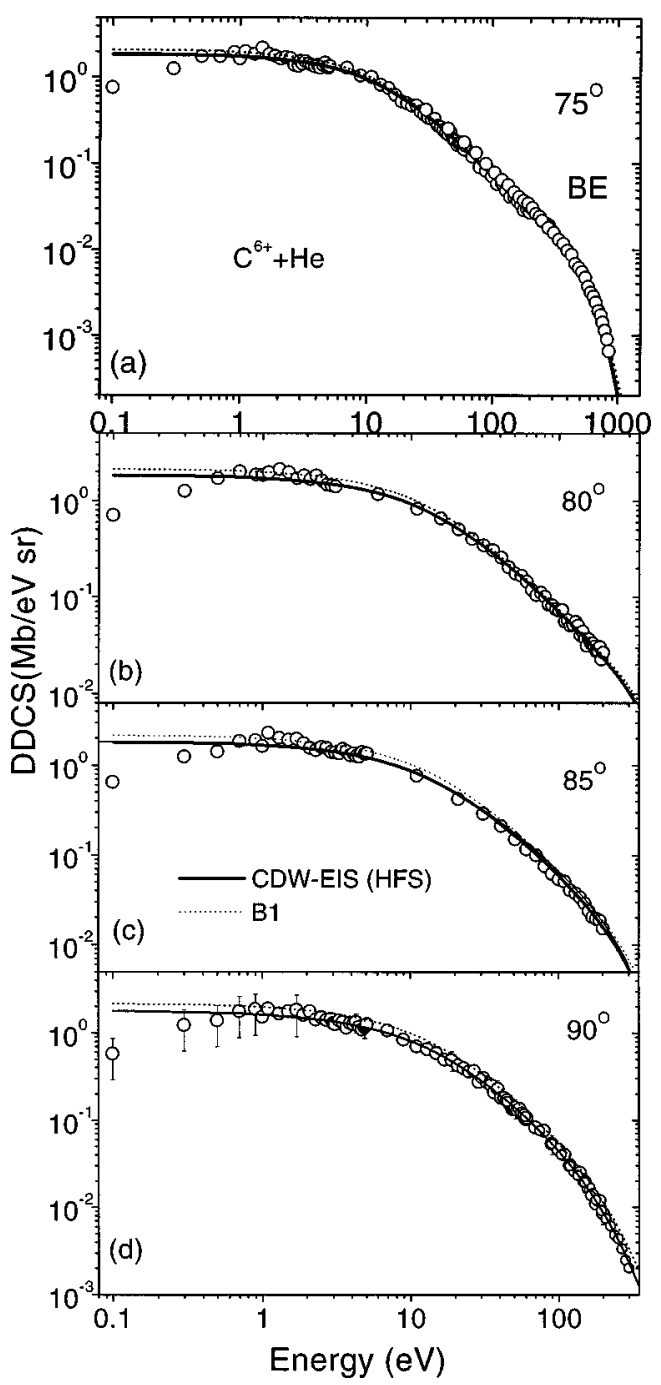

FIG. 2. Same as in Fig. 1 except for $\theta=75^{\circ}, 80^{\circ}, 85^{\circ}$, and $90^{\circ}$.

the B1 calculation with the HFS wave function for the ejected electron. The present calculations reproduce the results of the calculations in Ref. [3]. The calculations provide, in general, good agreement with the data below $10 \mathrm{eV}$. For higher energies $\left(\epsilon_{e} \geqslant 10 \mathrm{eV}\right)$ the agreement is poor for small forward angles $\left(\theta \leqslant 60^{\circ}\right)$ and for all the backward angles. The theory seems to reproduce the data quite well in the whole energy range for angles between $60^{\circ}$ and $90^{\circ}$.

The lowest-energy electrons are produced in a distant or soft collision and are too slow to follow the fast projectile. These soft electrons therefore would be less influenced by the projectile Coulomb field and hence their dynamics would be governed primarily by a one-center (target) Coulomb ionization. Hence it is not surprising that the B1 which explains the one-center emission quite well, gives good agreement below $10 \mathrm{eV}$. The deviation of B1 calculations for the extreme forward angles is caused by the postcollision interaction of the electrons with the projectile. These observations are consistent with the fact that the two-center mechanism of ionization and the postcollision effects, which are not included in the B1 calculations, are more important in the case of heavy-ion impact.

The CDW-EIS calculations with HFS [CDW-EIS(HFS)] and H-like [CDW-EIS(H)] wave functions are shown by

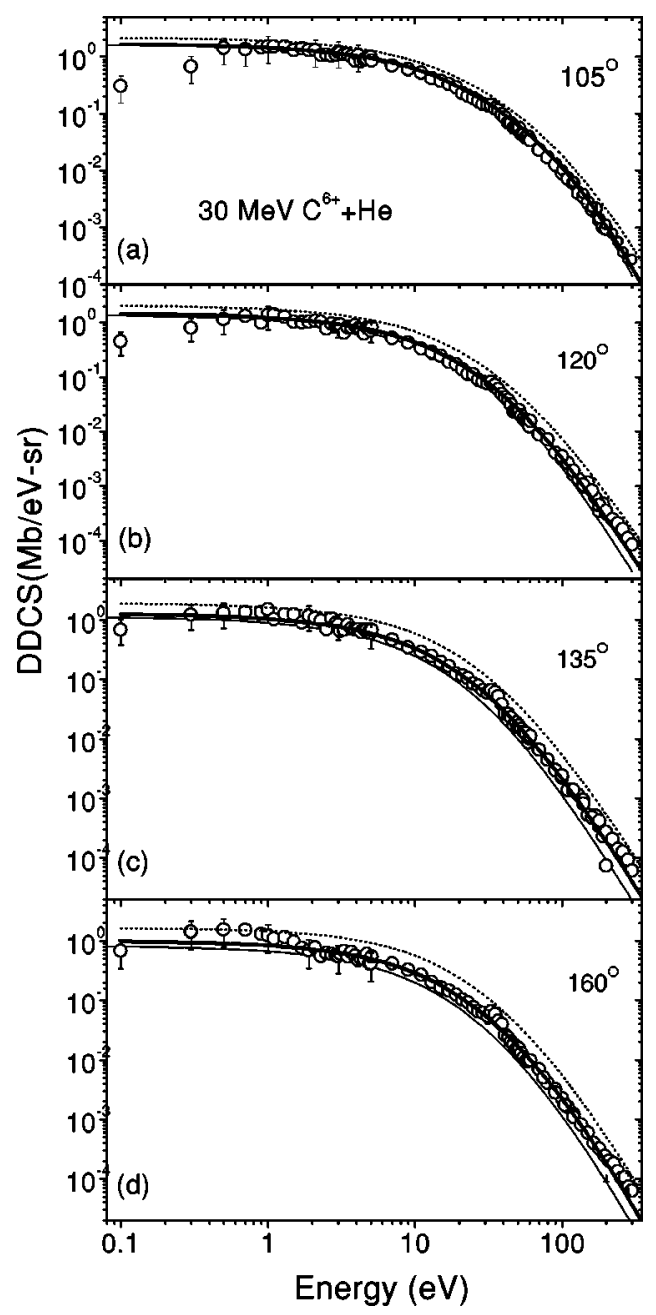

FIG. 3. Same as in Fig. 1 except for $\theta=105^{\circ}, 120^{\circ}, 135^{\circ}$, and $160^{\circ}$.

thick and thin solid lines, respectively. The CDW-EIS $(H)$ approximation provides good agreement with the data over the whole energy range except for small forward angles ( $\theta$ $\left.\leqslant 45^{\circ}\right)$ and large backward angles $\left(\theta \geqslant 120^{\circ}\right)$. For example, at $15^{\circ}$ and $30^{\circ}$ the theory [CDW-EIS(H)] falls slightly below the data above $10 \mathrm{eV}$ and underestimates the data throughout the whole energy range for $160^{\circ}$. It can be seen that the CDW-EIS calculations, using HFS wave functions to a large extent, reduce these discrepancies for both the forward and backward angles. In fact, the CDW-EIS(HFS) approximation provides excellent agreement with the data over the entire energy range for all the angles except for $\theta \leqslant 45^{\circ}$, for which the calculations fall about $30 \%$ below the data above $100 \mathrm{eV}$. Between $45^{\circ}$ and $120^{\circ}$ the CDW-EIS(H) and CDWEIS(HFS) approximations give almost identical results and provide an impressive agreement with the data over the whole energy range $(0.5-300 \mathrm{eV})$. Therefore, CDW-EIS $(\mathrm{H})$ calculations are not shown for $45^{\circ} \leqslant \theta \leqslant 90^{\circ}$. The two calculations differ for $\theta \leqslant 45^{\circ}$ and cross over around $10 \mathrm{eV}$, whereas the CDW-EIS $(\mathrm{H})$ result falls below the CDWEIS(HFS) result for large backward angles.

\section{B. Angular distributions at a fixed energy}

Although excellent agreement with the CDW-EIS(HFS) and CDW-EIS(H) approximations is observed in the energy 
TABLE I. Measured double-differential cross sections in units of Mb/eV sr for different angles. For errors see the text. Numbers in brackets denote multiplicative powers of 10 .

\begin{tabular}{|c|c|c|c|c|c|c|c|c|c|c|c|c|}
\hline$\varepsilon(\mathrm{eV})$ & $15^{\circ}$ & $30^{\circ}$ & $45^{\circ}$ & $60^{\circ}$ & $75^{\circ}$ & $80^{\circ}$ & $85^{\circ}$ & $90^{\circ}$ & $105^{\circ}$ & $120^{\circ}$ & $135^{\circ}$ & $160^{\circ}$ \\
\hline 0.1 & 1.07 & 0.47 & 0.51 & 0.80 & 0.76 & 0.70 & 0.64 & 0.58 & 0.30 & 0.45 & 0.68 & .68 \\
\hline 0.3 & 1.69 & 0.97 & 0.95 & 1.22 & 1.26 & 1.25 & 1.25 & 1.23 & 0.66 & 0.80 & 1.21 & 1.40 \\
\hline 0.5 & 1.68 & 1.03 & 1.25 & 1.84 & 1.73 & 1.70 & 1.42 & 1.38 & 1.42 & 1.14 & 1.30 & 1.55 \\
\hline 1 & 1.45 & 1.47 & 1.54 & 1.82 & 1.98 & 1.90 & 1.82 & 1.80 & 1.47 & 1.37 & 1.25 & 1.20 \\
\hline 3 & 1.40 & 1.17 & 1.19 & 1.54 & 1.54 & 1.41 & 1.38 & 1.28 & 1.16 & 0.87 & 0.82 & 0.55 \\
\hline 7 & 0.96 & 1.09 & 1.16 & 1.35 & 1.31 & 1.16 & 1.14 & 1.06 & 0.70 & 0.52 & 0.46 & 0.41 \\
\hline 11 & 0.87 & 0.88 & 0.92 & 1.02 & 1.00 & 0.82 & 0.77 & 0.693 & 0.51 & 0.333 & 0.29 & 0.265 \\
\hline 21 & 0.43 & 0.48 & 0.47 & 0.58 & 0.50 & 0.50 & 0.42 & 0.42 & 0.227 & 0.136 & 0.116 & 0.103 \\
\hline 30 & 0.25 & 0.27 & 0.300 & 0.361 & 0.39 & 0.34 & 0.29 & 0.28 & 0.143 & 0.079 & 0.62 & 0.58 \\
\hline 45 & 0.13 & 0.15 & 0.158 & 0.200 & 0.235 & 0.21 & 0.18 & 0.166 & 0.067 & 0.0306 & 0.0225 & 0.020 \\
\hline 60 & 0.079 & 0.082 & 0.090 & 0.133 & 0.160 & 0.147 & 0.12 & 0.110 & 0.0346 & 0.0123 & 0.0102 & 0.0098 \\
\hline 75 & 0.050 & 0.052 & 0.060 & 0.091 & 0.119 & 0.109 & 0.087 & 0.075 & 0.0200 & 0.079 & 0.0054 & 0.0054 \\
\hline 90 & 0.033 & 0.0325 & 0.041 & 0.065 & 0.090 & 0.082 & 0.061 & 0.053 & 0.0125 & 0.0041 & 0.0030 & 0.00316 \\
\hline 120 & 0.017 & 0.0173 & 0.0220 & 0.040 & 0.062 & 0.053 & 0.042 & 0.030 & 0.0059 & 0.00162 & 0.00135 & 0.00121 \\
\hline 150 & 0.010 & 0.0113 & 0.0137 & 0.027 & 0.044 & 0.038 & 0.030 & 0.019 & 0.0027 & 0.00115 & $5.23[-4]$ & $4.76[-4]$ \\
\hline 180 & 0.0063 & 0.00717 & 0.0085 & 0.0187 & 0.033 & 0.0280 & 0.0194 & 0.012 & 0.00133 & $3.94[-4]$ & $3.86[-4]$ & $3.60[-4]$ \\
\hline 210 & 0.0043 & 0.0053 & 0.0072 & 0.0150 & 0.0307 & & & 0.0073 & $2.9[-4]$ & $2.95[-4]$ & $2.37[-4]$ & $1.83[-4]$ \\
\hline 240 & 0.0031 & 0.00394 & 0.0051 & 0.0114 & 0.0244 & & & 0.0047 & $2.05[-4]$ & $2.05[-4]$ & $1.39[-4]$ & $1.07[-4]$ \\
\hline 270 & 0.0022 & 0.00287 & 0.0038 & 0.0095 & 0.0201 & & & 0.0033 & $1.35[-4]$ & $1.35[-4]$ & $1.07[-4]$ & $9.13[-4]$ \\
\hline 300 & 0.00164 & 0.00215 & 0.00307 & 0.0084 & 0.0167 & & & 0.0020 & $8.44[-5]$ & $8.44[-5]$ & $5.98[-5]$ & $1.03[-5]$ \\
\hline
\end{tabular}

dependence of the DDCS data, the comparison of the angular distribution data with the calculations may provide a deeper insight. In Fig. 4 we show the angular distributions for five different electron energies. The distributions peak at $75^{\circ}$ for all the energies.

For higher-energy electrons the distributions gradually become more peaked around $75^{\circ}$. Similar observations also have been reported before. The difference in the shape of the distributions for low- and high-energy electrons has been discussed in light of the binary nature of collisions [3]. The distributions are asymmetric about the peak, i.e., the cross sections at forward angles are much larger than those for backward angles.

For the lowest energy $(1 \mathrm{eV})$, the B1 calculation agrees with the data for forward angles but is too high by about $25 \%$ for the backward angles. The CDW-EIS(HFS) and CDW-EIS(H) approximations overestimate the data (for 1 $\mathrm{eV}$ ) by about $20 \%$ and $30 \%$, respectively, for extreme forward angles. For the largest backward angle the CDWEIS(HFS) and CDW-EIS(H) approximations give too-low cross sections compared to the data by about $30 \%$ and $40 \%$, respectively. These discrepancies are within the absolute errors, yet could be indicative of the failure of the theory for such low-energy electrons. The distribution at this energy is shifted slightly forward compared to the B1 prediction and may imply the presence of a very weak two-center effect even for soft collisions. Both of the CDW-EIS calculations predict an even larger forward shifting $\left(45^{\circ}\right)$ of the distribution that is not observed in the data. Since the shifting forward is indicative of the TCEE, it may be concluded that the CDW-EIS approximation overpredicts the two-center effects and the postcollision interactions for very soft collisions. At slightly higher energy (10 and $50 \mathrm{eV})$ the data show little additional shifting compared to the B1 calculations. The cross sections are enhanced in the forward direction and depleted in the backward direction compared to the B1 predictions and may be explained as the deflection of the electrons in the forward direction due to the postcollision interaction with the projectile. The CDW-EIS(H) approximation underestimates the data (by about $25-50 \%$ ) at these energies (10 and $50 \mathrm{eV}$ ) for both the extreme forward and large backward angles, whereas the CDW-EIS(HFS) approximation provides almost perfect agreement with the data for all the angles. For the higher energies (150 and $300 \mathrm{eV})$ the sharp peak is reproduced by the $\mathrm{B} 1$ and both of the CDW-EIS theories. A large portion of the these high-energy $(150$ and $300 \mathrm{eV})$ electrons that are emitted around $70^{\circ}$ (i.e., at the peak of the angular distribution) come from the binary encounter process [see the BE hump in Fig. 2(a)], which is a pure two-body process and hence the B1 also explains the data. However, the same energy electrons emitted in smaller angles $\left(\theta \leqslant 60^{\circ}\right)$ are separated from the BE peak [see Figs. 1(c) and $1(\mathrm{~d})]$ and are mostly contributed to by the three-body ionization process. The CDW-EIS(H) calculations largely underestimate the data for extreme forward and large backward angles, but the CDW-EIS(HFS) calculation provides a much better explanation of the data. Note that for large backward angles the CDW-EIS(HFS) approximation underestimates the data (at $300 \mathrm{eV}$ ), but the B1 reproduces it quite well. These observations are not understood and need to be studied in more detail.

\section{Angular asymmetry}

We express the angular distribution of the electrons emitted into the solid angle $d \Omega$ with energy $\varepsilon=k^{2} / 2$ by the expression [21] 


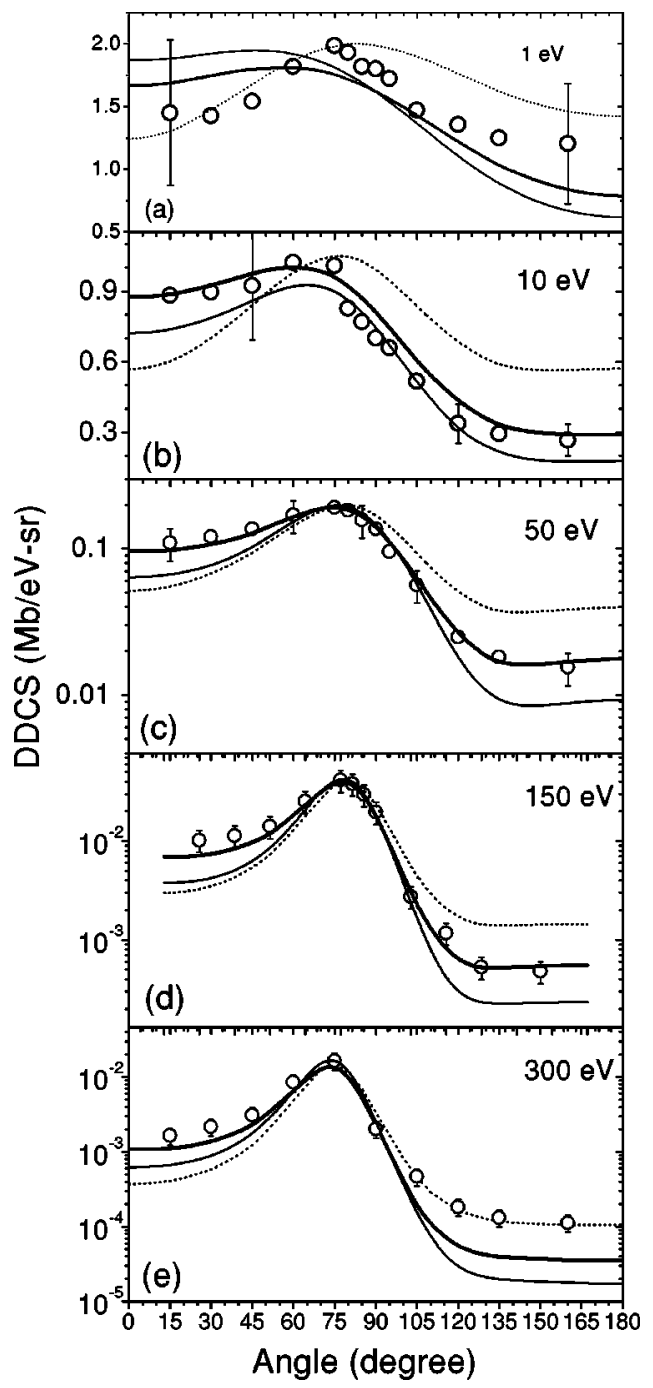

FIG. 4. Angular distribution of electrons for a fixed energy. The thick solid, thin solid, and dotted lines represent the CDWEIS(HFS), CDW-EIS(H), and B1 calculations, respectively.

$$
S(\theta)=\frac{d^{2} \sigma}{d \varepsilon d \Omega}=\sum \beta_{L}(k) P_{L}(\cos \theta),
$$

where $P_{L}$ is the Legendre polynomial. The asymmetry parameter is defined as

$$
\alpha(k)=\frac{S(0)-S(\pi)}{S(0)+S(\pi)}=\frac{\sum_{j} \beta_{2 j+1}(k)}{\sum_{j} \beta_{2 j}(k)} \approx \frac{\beta_{1}(k)}{\beta_{0}(k)+\beta_{2}(k)} .
$$

Although $S(0)$ and $S(\pi)$ were not measured, they could be deduced by extrapolating the angular distributions since the distributions vary smoothly near $0^{\circ}$ and $180^{\circ}$. It may be seen from Fig. 5 that $\alpha(k)$ is very small $(\approx 0.1)$ in the zeroenergy limit and increases with the electron energy. The B1 predicts the value of $\alpha(k)$ lower than the data, while the CDW-EIS(HFS) approximation is in much better agreement. A large forward-backward asymmetry was observed by Suaréz et al. [6] for low-energy electrons emitted in 106-keV $p+\mathrm{Ne}$ collisions and was interpreted as due to the two-

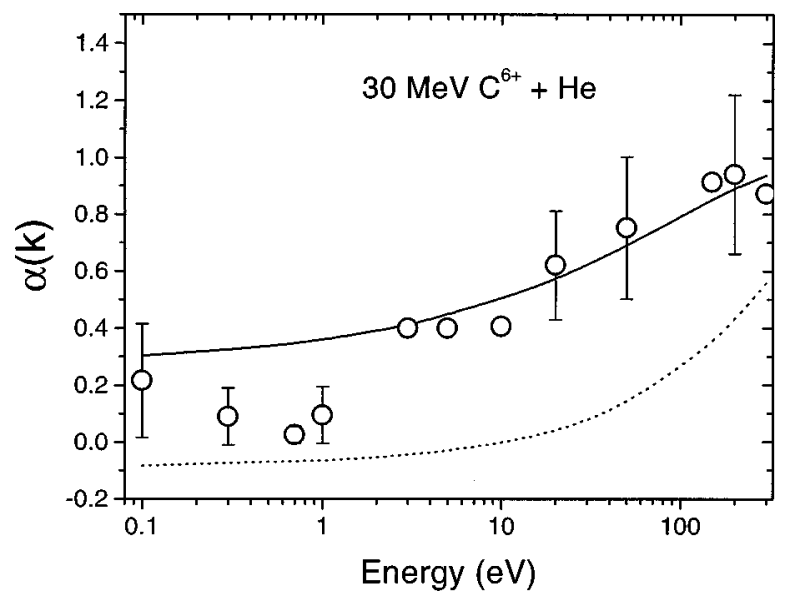

FIG. 5. Forward-backward asymmetry parameter $\alpha(k)$ as a function of electron energy. The solid and dotted lines denote the CDW-EIS(HFS) and B1 calculations, respectively.

center electron (TCE). However, there was a large discrepancy between the observed $\alpha(k)$ values and the CDWEIS(HFS) calculations for $\varepsilon \leqslant 10 \mathrm{eV}$. Fainstein et al. [21] have shown that apart from the TCE, the forward-backward asymmetry can also result if the ionized electron moves in a non-Coulomb field, as in the case of any multielectron target (such as $\mathrm{He}$, in the present case). As a result, the B1 also gives forward-backward asymmetry, i.e., nonzero $\alpha$, for $\varepsilon$ $\rightarrow 0$. In the present case the $\alpha(k)$ 's from the B1 are very small, which was not the case for $100-\mathrm{keV} p+\mathrm{Ne}$ [21] studied earlier. So for the present system the TCE is the most important contributer to the angular asymmetry of the lowenergy electron emission. For higher electron energies $(\varepsilon$ $\geqslant 50 \mathrm{eV}$ ) the B1 and CDW-EIS(HFS) curves are parallel or approach each other weakly with increasing energy and this may mean that the role of two-center effects is constant or decreases slightly with increasing electron energies.

\section{Single-differential cross sections}

The singly differential distributions $d \sigma / d \Omega$ and $d \sigma / d \varepsilon$ were derived by performing numerical integration of the energy or angular distributions of the DDCSs, respectively. Figure 6 shows the $d \sigma / d \varepsilon$ as a function of $\varepsilon$ (see also Table II). Excellent agreement between the data (circles) and both of the CDW-EIS calculations throughout the whole energy range is observed. Interestingly, there is no appreciable difference between the CDW-EIS(HFS) and CDW-EIS(H) calculations. This is because the forward and backward angles, where the largest discrepancies have been observed between the two calculations, are less important in the integrated cross sections. Overall agreement with the B1 calculation is also good except below $10 \mathrm{eV}$, for which the theory overestimates the data by about $20-25 \%$. We have also shown (square) similar data (taken from Ref. [15]) for the ionization of $\mathrm{H}_{2}$ by the same projectile. The cross sections in the case of $\mathrm{H}_{2}$ are larger compared to those for the He target as expected, based on the binding-energy considerations. However, the differences in the cross sections are large only for low-energy $(<10 \mathrm{eV})$ electrons. Above $50 \mathrm{eV}$ the cross sections for $\mathrm{H}_{2}$ and $\mathrm{He}$ are almost the same and imply that a majority of the higher-energy electrons $(\varepsilon \geqslant 50 \mathrm{eV})$ may 


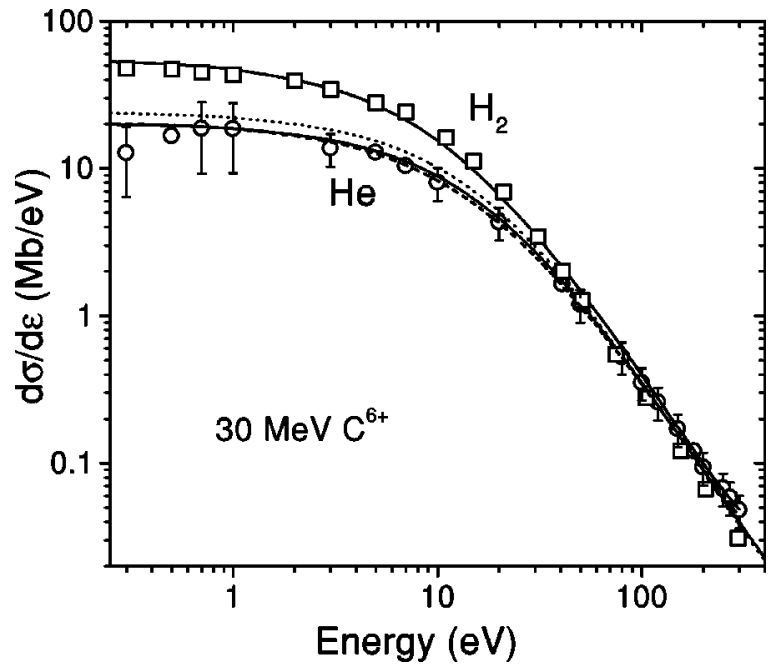

FIG. 6. Single-differential cross sections $\left(d \sigma / d \varepsilon_{e}\right)$ for $\mathrm{C}^{6+}+\mathrm{He}$ (circles) as a function of electron energy. The squares represent the data for 2.5-MeV/nucleon $\mathrm{C}^{6+}+\mathrm{H}_{2}$ (from Tribedi et al. [15]). The solid, dashed, and dotted lines represent the CDW-EIS(HFS), CDW-EIS(H), and B1 calculations.

originate from violent, close collisions so that the emission probability is almost independent of the initial binding energy. The angular distributions of the single differential cross sections $(d \sigma / d \Omega)$ are shown in Fig. 7 (see Table III). The peak observed at $70^{\circ}$ is consistent with the angular distributions of the DDCSs as discussed before. The B1 gives a reasonable explanation of the data, although the CDWEIS(HFS) approximation provides better agreement. The CDW-EIS(H) approximation underestimates the data at large backward angles by about 25 to $40 \%$. The two CDW-EIS models start to differ above $105^{\circ}$ and the difference becomes as large as $40 \%$ for $160^{\circ}$.

The total cross sections may be calculated by integrating the SDCSs in either Fig. 6 or 7. The results of these integrations were $323 \mathrm{Mb}$ and $317 \mathrm{Mb}$, respectively, and are for electron energies between 0.1 and $300 \mathrm{eV}$ and emission angle between $15^{\circ}$ and $160^{\circ}$. The CDW-EIS(HFS) approximation predicts $320 \mathrm{Mb}$ for the same limits and hence is in excellent agreement with the data. The $\mathrm{B} 1$ gives $358 \mathrm{Mb}$ as the integrated cross section. Inclusion of all the angles $\left(0^{\circ}-180^{\circ}\right)$ and higher energies $(0-5000 \mathrm{eV})$ increases the CDW-EIS(HFS) value to $347 \mathrm{Mb}$.

TABLE II. Single-differential cross sections $(d \sigma / d \varepsilon)$ for some selected electron energies $(\varepsilon)$ in units of $\mathrm{Mb} / \mathrm{eV}$. Typical errors are about $25 \%$ above $5 \mathrm{eV}$ (see the text).

\begin{tabular}{ccrccl}
\hline \hline$\varepsilon$ & $d \sigma / d \varepsilon$ & $\varepsilon$ & $d \sigma / d \varepsilon$ & $\varepsilon$ & $d \sigma / d \varepsilon$ \\
\hline 0.1 & 7.06 & 7 & 10.5 & 120 & 0.26 \\
0.3 & 12.8 & 10 & 8.05 & 150 & 0.172 \\
0.5 & 16.68 & 20 & 4.35 & 180 & 0.121 \\
0.7 & 18.75 & 40 & 1.65 & 200 & 0.0944 \\
1 & 18.64 & 50 & 1.2 & 250 & 0.068 \\
3 & 13.76 & 80 & 0.53 & 270 & 0.0592 \\
5 & 12.87 & 100 & 0.354 & 300 & 0.048 \\
\hline \hline
\end{tabular}

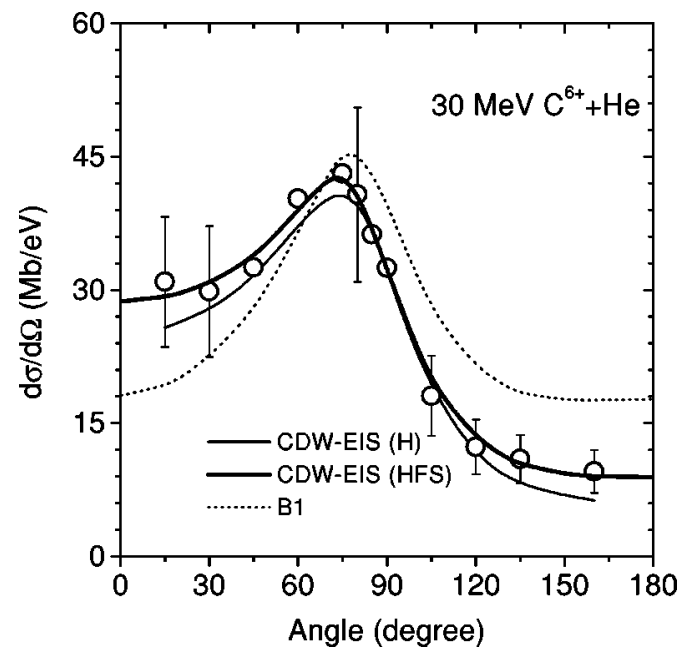

FIG. 7. Single-differential cross sections $\left(d \sigma / d \Omega_{e}\right)$ as a function of electron emission angle. The symbols have the same meaning as in Fig. 6.

\section{E. Comparison between the DDCSs for $\mathrm{He}$ and $\mathrm{H}_{2}$}

We show in Fig. 8 the ratio $(R)$ of the DDCSs for the He and $\mathrm{H}_{2}$ targets measured at $60^{\circ}$. The expected value of $R$ is estimated to be approximately equal to 0.4 (dashed line) based on the fact that the DDCS is inversely proportional to the square of the initial binding energy. The binding energies for $\mathrm{H}_{2}$ and $\mathrm{He}$ targets are assumed to be those for the unperturbed states, i.e., 0.57 and 0.903 a.u., respectively. Interestingly, the observed ratio is not a constant but rather changes drastically with the electron energy. It may be seen that for the lowest-energy electrons the experimental data fall near the dotted line. This is consistent with the fact that the lowest-energy electrons are mostly emitted in the distant or large impact parameter collisions in which case the initial state of the target atom is not perturbed much and hence the assumption of the initial unperturbed binding energies holds true. As the energy increases, the ratio increases to approximately 2.5. For the binary encounter electrons the ratio decreases again. At the binary encounter the projectile suffers a close collision and a large amount of momentum is transferred to the electron. Therefore, the cross section for electron emission at the BE peak is almost independent of the binding energy and the value of $R$ should be nearly one, as can be seen from the data. Beyond the BE region the ratio increases again. Such a structure is also reproduced by the

TABLE III. Single-differential cross sections $(d \sigma / d \Omega)$ for different angles $(\theta)$ in units of $\mathrm{Mb} / \mathrm{sr}$. Typical errors are about $25 \%$.

\begin{tabular}{ccccc}
\hline \hline$\theta$ & $15^{\circ}$ & $30^{\circ}$ & $45^{\circ}$ & $60^{\circ}$ \\
\hline$d \sigma / d \Omega$ & 30.9 & 29.8 & 32.5 & 40.2 \\
$\theta$ & $75^{\circ}$ & $80^{\circ}$ & $85^{\circ}$ & $90^{\circ}$ \\
$d \sigma / d \Omega$ & 43.1 & 40.7 & 36.2 & 32.5 \\
$\theta$ & $105^{\circ}$ & $120^{\circ}$ & $135^{\circ}$ & $160^{\circ}$ \\
$d \sigma / d \Omega$ & 18.1 & 12.3 & 10.95 & 9.55 \\
\hline \hline
\end{tabular}




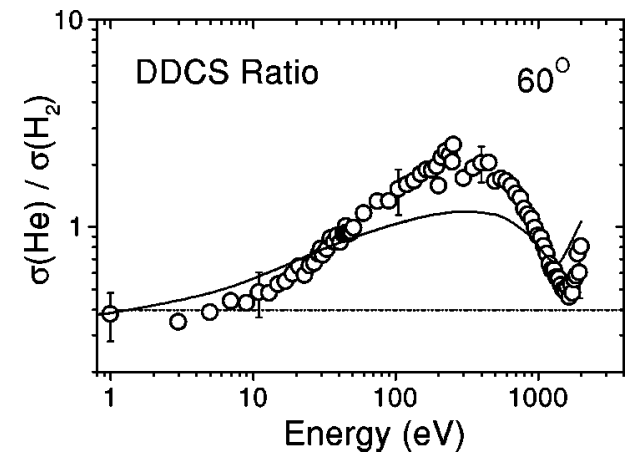

FIG. 8. Ratio of the DDCS for He to that of $\mathrm{H}_{2}$ for $\theta=60^{\circ}$. The solid line is the calculation using HFS wave functions for $\mathrm{He}$ and $\mathrm{H}$-like wave function for $\mathrm{H}_{2}$. For the dotted line, see the text.

CDW-EIS theory, although there are some differences. For these calculations the HFS and H-like wave functions were used for $\mathrm{He}$ and $\mathrm{H}_{2}$, respectively. The two-center effect cannot cause such a structure in the DDCS ratio since both of the targets are influenced in a similar way by the TCE. This is also evident from the observation that the B1 reproduces the structure in $R$ quite well (not shown). The difference in the initial state momentum distribution of the electrons in two different atoms is believed to be responsible for such a structure. The electron-electron correlation may also play a role.

\section{CONCLUSIONS}

We have measured the energy and angular distributions of the double differential cross sections of low-energy (0.1-300 $\mathrm{eV})$ electron emission in ion-atom ionization for $2.5-\mathrm{MeV} /$ nucleon $\mathrm{C}^{6+}+\mathrm{He}$. A detailed comparison between the DDCS for $\mathrm{H}_{2}$ and $\mathrm{He}$ is presented. The two-center effect has been explored in detail. The CDW-EIS(HFS) approximation provides excellent agreement with the data, although some discrepancies exist. The CDW-EIS(H) approximation shows several disagreements for large backward angles and extreme forward angles. However, both calculations are based on the independent electron approximation where the role of electron-electron interaction is neglected. It is not clear whether the electron-electron interaction plays a role for the observed small discrepancy with the theory. The forwardbackward asymmetry parameters were deduced from the observed angular distributions for different electron energies. From a comparison with the CDW-EIS(HFS) and B1 calculations, it was concluded that, for the present system, the forward-backward asymmetry is mainly caused by the twocenter effect. The measurements of the projectile energy dependence of the electron DDCS also should be addressed and work in this direction is in progress.

\section{ACKNOWLEDGMENT}

This work was supported by the Division of Chemical Sciences, Office of Basic Energy Sciences, Office of Energy Research, U.S. Department of Energy.
[1] G. B. Crooks and M. E. Rudd, Phys. Rev. Lett. 25, 1599 (1970).

[2] K. G. Harrison and M. Lucas, Phys. Lett. 33A, 149 (1970).

[3] Steven T. Manson, L. H. Toburen, and N. Stolterfoht, Phys. Rev. A 12, 60 (1975).

[4] M. E. Rudd, L. H. Toburen, and N. Stolterfoht, At. Data Nucl. Data Tables 18, 413 (1976).

[5] M. E. Rudd, Y. Kim, D. Madison, and T. Gay, Rev. Mod. Phys. 64, 441 (1992).

[6] S. Suárez, C. Garibotti, W. Meckbach, and G. Bernardi, Phys. Rev. Lett. 70, 418 (1993).

[7] M. W. Gealy, G. W. Kerby III, Y.-Y. Hsu, and M. E. Rudd, Phys. Rev. A 51, 2247 (1995).

[8] G. W. Kerby III, M. W. Gealy, Y.-Y. Hsu, M. E. Rudd, O. R. Schultz, and C. O. Reinhold, Phys. Rev. A 51, 2256 (1995).

[9] Y.-Y. Hsu, M. W. Gealy, G. W. Kerby III, and M. E. Rudd, Phys. Rev. A 53, 297 (1996); Y.-Y. Hsu, M. W. Gealy, G. W. Kerby III, M. E. Rudd, D. R. Schultz, and C. O. Reinhold, ibid. 53, 303 (1996).

[10] D. H. Lee, P. Richard, T. J. M. Zouros, J. M. Sanders, J. L. Shinpaugh, and H. Hidmi, Phys. Rev. A 41, 4816 (1990).

[11] J. O. P. Pedersen, P. Hvelplund, A. Petersen, and P. Fainstein, J. Phys. B 24, 4001 (1991).
[12] R. D. DuBois, Phys. Rev. A 50, 364 (1994).

[13] C. Liao, P. Richard, S. R. Grabbe, C. P. Bhalla, T. J. M. Zouros, and S. Hagmann, Phys. Rev. A 50, 1328 (1994).

[14] N. Stolterfoht, H. Platten, G. Schiwietz, D. Schneider, L. Gulyás, P. D. Fainstein, and A. Salin, Phys. Rev. A 52, 3796 (1995).

[15] Lokesh C. Tribedi, P. Richard, D. Ling, Y. D. Wang, C. D. Lin, R. Moshammer, G. W. Kerby III, M. W. Gealy, and M. E. Rudd, Phys. Rev. A 54, 2154 (1996).

[16] D. S. F. Crothers and J. F. McCann, J. Phys. B 16, 3229 (1983).

[17] P. D. Fainstein, V. H. Ponce, and R. D. Rivarola, J. Phys. B 21, 287 (1988).

[18] P. D. Fainstein, V. H. Ponce, and R. D. Rivarola, J. Phys. B 24, 3091 (1991).

[19] E. Clementi and C. Roetti, At. Data Nucl. Data Tables 14, 177 (1974).

[20] L. Gulyás, P. D. Fainstein, and A. Salin, J. Phys. B 28, 245 (1995).

[21] P. D. Fainstein, L. Gulyás, F. Martin, and A. Salin, Phys. Rev. A 53, 3243 (1996).

[22] D. E. Golden and H. W. Bandel, Phys. Rev. 138, A14 (1965). 